\title{
Electron-Determined Nonuniform Carrier Distribution among InGaAsP Multiple Quantum Wells
}

Ching-Fuh LIN ${ }^{1,2}$, Bing-Ruey $\mathrm{WU}^{3}$ and Lih-Wen LAIH ${ }^{3}$

${ }^{1}$ Graduate Institute of Electro-Optical Engineering, National Taiwan University, Taipei, Taiwan, R.O.C.

${ }^{2}$ Department of Electrical Engineering, National Taiwan University, Taipei, Taiwan, R.O.C.

${ }^{3}$ Telecommunication Laboratory, Chunghua Telecom Co., Ltd., Yang-Mei, Taoyuan, Taiwan, R.O.C.

(Received October 30, 2002; accepted for publication April 14, 2003)

Experiments on laser diodes and superluminescent diodes with nonidentical InGaAsP multiple quantum wells (MQWs) show that quantum wells near the n-cladding layer could accumulate more carriers than those near the p-cladding layer, indicating that nonuniform carrier distribution is determined by electrons instead of holes. The electron-determined behavior is attributed to the thick separate-confinement heterostructure layer. This contrary observation to hole-determined nonuniform carrier distribution implies that carrier distribution among the MQWs could be engineered for desired purposes.

[DOI: $10.1143 / \mathrm{JJAP} .42 .5557]$

KEYWORDS: laser diodes, superluminescent diodes, multiple quantum wells, nonuniform carrier distribution, separate-confinement heterostructure layer

Carrier distribution in multiple quantum wells (MQWs) is an important issue for understanding the characteristics of quantum-well lasers. ${ }^{1)}$ Nonuniform carrier distribution among the MQWs had been theoretically predicted ${ }^{2,3)}$ and experimentally observed. ${ }^{4-7)}$ In the past, because experiments showed that quantum wells (QWs) near the p-cladding layer accumulated more carriers than those near the ncladding layer, holes were deemed as the dominant carriers determining the nonuniform carrier distribution. In this work, we report that QWs near the n-cladding layer could also accumulate more carriers than those near the p-cladding layer. This contrary observation shows that electrons instead of holes could determine the behavior of nonuniform carrier distribution in some cases. This discovery suggests that carrier distribution among the MQWs could be engineered by making either electrons or holes the dominant carriers.

Mirror-imaged nonidentical MQWs were used in the experiments. The MQW structure has three $60 \AA$ $\mathrm{In}_{0.67} \mathrm{Ga}_{0.33} \mathrm{As}_{0.72} \mathrm{P}_{0.28} \quad \mathrm{QWs}$ and two $87 \AA \mathrm{In}_{0.53} \mathrm{Ga}_{0.47} \mathrm{As}$ QWs, separated by a $150 \AA \operatorname{In}_{0.86} \mathrm{Ga}_{0.14} \mathrm{As}_{0.3} \mathrm{P}_{0.7}$ barrier. ${ }^{8)}$ According to the calculation using the Luttinger-Kohn method, ${ }^{9,10)}$ the $60 \AA \operatorname{In}_{0.67} \mathrm{Ga}_{0.33} \mathrm{As}_{0.72} \mathrm{P}_{0.28}$ QWs and the $87 \AA \mathrm{In}_{0.53} \mathrm{Ga}_{0.47}$ As QWs have their first quantized energies corresponding to $\lambda=1.3 \mu \mathrm{m}$ and $\lambda=1.54 \mu \mathrm{m}$, respectively. Substrates 04291 and 04292 have two $87 \AA$ QWs near the ncladding layer and the p-cladding layer, respectively. The layer of separate confinement heterostructure $(\mathrm{SCH})$ is $120 \mathrm{~nm}$ thick. The layer structure of 04291 is the same as the one reported in ref. 8. Fabry-Perot laser diodes (LDs), tilted-stripe, and bent-waveguide superluminescent diodes $(\mathrm{SLDs})^{8)}$ were fabricated on those substrates with standard processing techniques. The devices all had a $6-\mu \mathrm{m}$-wide ridge waveguide. Device lengths of about $500 \mu \mathrm{m}$ and $700 \mu \mathrm{m}$ were obtained. No coating was applied to the device facets. LDs fabricated on substrates 04291 and 04292 are simply named as 04291 LDs and 04292 LDs, respectively. SLDs are also named in a similar manner.

Both 04291 and 04292 LDs have a threshold current of approximately $55 \mathrm{~mA}$. Figure 1 shows the lasing spectra of the 04291 and 04292 LDs. 04291 LDs oscillate at approximately $1500 \mathrm{~nm}$, which is mainly contributed from the $87 \AA$ QWs. In contrast, 04292 LDs have a lasing wavelength around $1415 \mathrm{~nm}$, contributed from both the $60 \AA \mathrm{QWs}$ and the $87 \AA$ QWs. The comparison of the lasing wavelengths

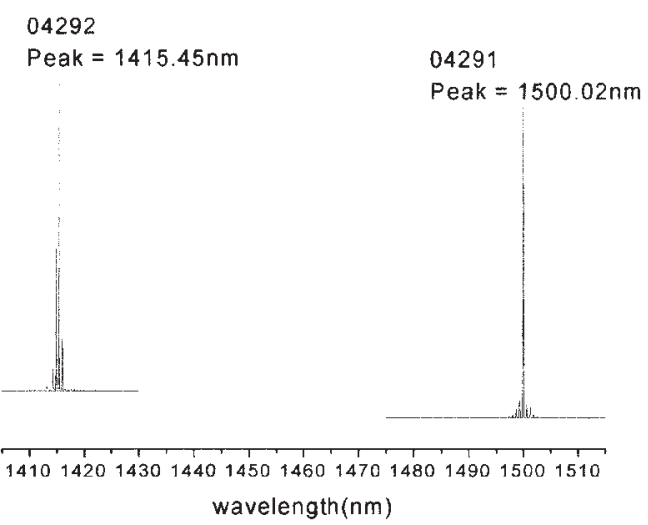

Fig. 1. The lasing spectra of 04291 and 04292 LDs.

from the two types of LDs indicates that the QWs near the ncladding layer trap more carriers than those near the pcladding layer. Because electrons are injected from the ncladding layer, the carrier distribution is determined by electrons instead of holes in this case. To confirm the electron-determined behavior further, the SLDs were also measured. Because SLDs have no threshold behavior or resonance modes, their emission spectra should directly reflect the carrier distribution in the corresponding QWs. Figure 2 shows the emission spectra of the 04291 and 04292 SLDs. For 04291 SLDs, emission at a low injection current occurs at the wavelength corresponding to the $n=1$ transition in the $87 \AA$ QWs, which are close to the ncladding layer. For 04292 SLDs, the emission occurs at the wavelength corresponding to $60 \AA \mathrm{QWs}$, which are also close to the n-cladding layer. More than 10 devices of each type were measured. The $500 \mu \mathrm{m}$ and $700 \mu \mathrm{m}$ LDs showed similar behaviors, and the $500 \mu \mathrm{m}$ and $700 \mu \mathrm{m}$ tilted-stripe, bent-waveguide SLDs all behave similarly. The measured spectra show that more carriers are trapped in the QWs near the n-cladding layer instead of the p-cladding layer. Therefore, the electron-determined carrier distribution is clearly evidenced.

The discrepancy between our experiment and past observation raises the necessity of further discussion regarding dominant carriers that determine carrier distribution among MQWs. The past observation of hole-determined nonuniform carrier distribution is mainly explained by the 
(a)

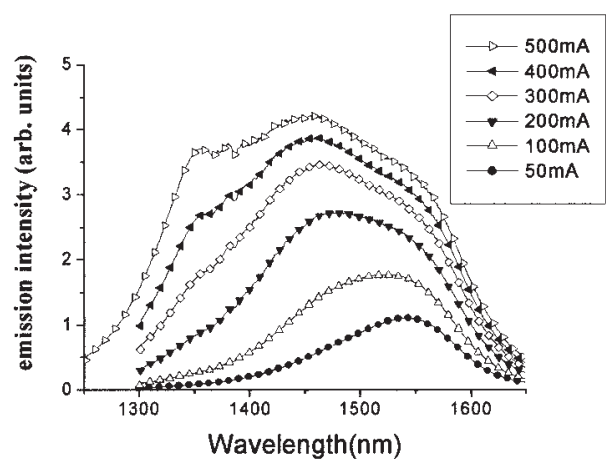

(b)

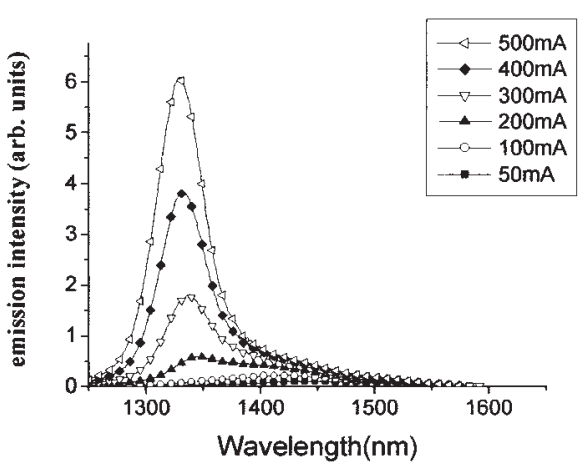

Fig. 2. The emission spectra: (a) 04291 SLDs; (b) 04292 SLDs.

slow movement of holes, ${ }^{2,3,5,7)}$ leading to hole localization. Then, the highly mobile electrons follow the hole distribution. Such an explanation is based on the assumption that the electrons captured in the QWs could easily escape and redistribute among the MQWs. However, the escape probability is proportional to $\exp [-\Delta E / k T]{ }^{5)}$ where $\Delta E$ is the barrier height of QWs. For $k T \ll \Delta E$, both electrons and holes cannot easily escape from QWs. Therefore, not only hole localization but also electron localization could occur. Carrier distribution is not solely determined by holes.

To explain both our new discovery and previous observations, we assert that the type of carriers that first arrive at the QWs will determine the final carrier distribution. At the beginning of carrier injection, electrons move from the ncladding layer, while holes move from the p-cladding layer. If the electrons arrive at the QWs earlier than the holes, they will most likely be captured in the QWs nearest to the ncladding. At this time, if the holes are still far away from the QW region, then the electrons and holes are far apart. Therefore, the band tilt across the $\mathrm{SCH}$ and $\mathrm{QW}$ region is small. As a result, the small band tilt makes it difficult for the electrons to escape. Then the holes continue to move toward the QWs. When the holes are close to the QWs, the distance between the hole cluster and the electron cluster is very short. Thus, the band tilt is large. This indicates that the electrical field is strong and holes tend to be pulled toward electrons instead of being captured into QWs near the p- cladding. This mechanism causes holes to follow the electron distribution to achieve the condition of quasicharge neutrality. Therefore, electrons determine the nonuniform carrier distribution. On the other hand, if holes arrive at the QWs first, the nonuniform carrier distribution is determined by holes.

Whether electrons or holes first reside in QWs depends on the summation of their capture time and their diffusion time across the SCH layer. The capture time for electrons and holes is about $1 \mathrm{ps}\left(\tau_{\mathrm{cn}}\right)$ and $0.2 \mathrm{ps}\left(\tau_{\mathrm{cp}}\right)$, respectively. ${ }^{11,12)}$ The diffusion time for electrons and holes is given by $d_{\mathrm{n}}^{2} / 4 D_{\mathrm{n}}$ and $d_{\mathrm{p}}^{2} / 4 D_{\mathrm{p}}$, respectively, where $d_{\mathrm{n}}$ and $d_{\mathrm{p}}$ are the thickness of the SCH layer and $D_{\mathrm{n}}$ and $D_{\mathrm{p}}$ are the diffusion constant for electrons and holes, respectively. Because our layer structures have a very thick $\mathrm{SCH}$ layer, the hole diffusion time, $d_{\mathrm{p}}^{2} / 4 D_{\mathrm{p}}$, is about $11 \mathrm{ps}$, which is much longer than the sum of the electron diffusion time and the electron capture time. Thus, electrons reside in the QWs before holes, leading to carrier distribution determined by electrons. In contrast, the layer structure of the previous experiment ${ }^{5,6}$ has a comparatively thin $\mathrm{SCH}$ layer, so the nonuniform carrier distribution is determined by holes. ${ }^{5,6)}$

In conclusion, electron-determined nonuniform carrier distribution among MQWs is observed in LDs/SLDs fabricated on substrates with mirror-imaged nonidentical MQWs. The fabricated InGaAsP LDs/SLDs all demonstrate that QWs near the n-cladding layer trap more carriers than QWs near the p-cladding layer. To explain the observation, we assert that the first type of carriers residing in the QWs will determine the carrier distribution. In our design, the thick SCH layer results in a long diffusion time of holes, so electrons are first captured into QWs, causing the carrier distribution to be favored in QWs near the n-cladding layer.

This work is supported in part by the National Science Council, Taipei, Taiwan, R.O.C. under contract Nos. NSC892215-E-002-016 and NSC89-2112-M-002-034.

1) R. Nagarajan, T. Fukushima, S. W. Corzine and J. E. Bowers: Appl. Phys. Lett. 59 (1991) 1835.

2) A. Hangleiter, A. Grabmaier and G. Fuchs: Appl. Phys. Lett. 62 (1993) 2316.

3) N. Tessler and G. Eisenstein: IEEE J. Quantum Electron. 29 (1993) 1586.

4) K. Frojdh, S. Marcinkevicius, U. Olin, C. Silfvenius, B. Stalnacke and G. Landgren: Appl. Phys. Lett 69 (1996) 3695.

5) H. Yamazaki, A. Tomita and M. Yamaguchi: Appl. Phys. Lett. 71 (1997) 767.

6) B.-L Lee, C.-F. Lin, J.-W Lai and W. Lin: Electron. Lett. 34 (1998) 1230 .

7) M. J. Hamp, D. T. Cassidy, B. J. Robinson, Q. C. Zhao, D. A. Thompson and M. Davies: IEEE Photon. Technol. Lett. 10 (1998) 1380.

8) B.-R. Wu, C.-F. Lin, L.-W. Laih and T.-T. Shih: Electron. Lett. 36 (2000) 2093.

9) J. M. Luttinger and W. Kohn: Phys. Rev. 97 (1955) 869.

10) D. Ahn and S. L. Chuang: IEEE J. Quantum Electron. 26 (1990) 13.

11) M. Alam and M. Lundstrom: IEEE Photon. Technol. Lett. 6 (1994) 1418.

12) S. C. Kan, D. Vassilovski, T. C. Wu and K. Y. Lau: Appl. Phys. Lett. 61 (1992) 752. 